\title{
Le numérique, le risque de ne plus prévenir le non-recours
}

Héléna Revil, Philippe Warin Héléna Revil est chercheure associée au laboratoire de sciences sociales PACTE-CNRS/université Grenoble Alpes/Sciences Po Grenoble, et responsable scientifique de l'Observatoire des non-recours aux droits et services (Odenore) où elle dirige le programme sur le non-recours en santé. Ses travaux proposent une analyse du système de protection sociale et de santé à partir des rapports différenciés des personnes avec l'action sanitaire et sociale.

Philippe Warin est directeur de recherche CNRS au laboratoire PACTE et cofondateur de l'Odenore. Son programme de recherche a pour but de développer une analyse sociopolitique de la réception des politiques par les publics.

Le programme «Action publique 2022 » lancé par le Premier ministre le 13 octobre 2017 vise à transformer l'administration sur trois points : améliorer la qualité des services publics, offrir un environnement de travail modernisé aux professionnels et maîtriser les dépenses publiques en optimisant les moyens. Son ambition générale est de repenser le modèle de l'action publique en interrogeant en profondeur les métiers et les modes d'intervention. Cette transformation doit être menée en particulier au regard de la " révolution numérique », pour atteindre un objectif de «100\% de services publics dématérialisés à horizon $2022 »$. Cet objectif suppose de réunir de nombreuses conditions. Surtout, il interroge sur les possibles conséquences induites par des relations administratives qui seraient largement, sinon totalement, dématérialisées. En effet, si ce programme est mis en œuvre, il ne sera plus question dans un proche avenir de réaliser les démarches administratives, quelles qu'elles soient, sans utiliser d'abord, sinon uniquement, un ordinateur afin d'accomplir les différentes tâches nécessaires : dépôts en ligne de demandes et de documents; transferts de données ; prises de rendez-vous ; suivis des dossiers de demandes, etc.

Les inquiétudes que suscite un tel changement sont suffisamment développées pour qu'il ne soit pas nécessaire de les évoquer à nouveau ici. De nombreux dossiers documentent le sujet ${ }^{1}$ et des rapports alertent les pouvoirs publics sur les conséquences irrattrapables d'une dématérialisation totale ${ }^{2}$. L'interrogation commune est la suivante : que peut-il se passer dès lors que les relations administratives deviennent immatérielles ? Cette question est ancienne. Elle renvoie notamment à toute une réflexion théorique sur "l'informatisation de la société » qui a très tôt ouvert des débats sur les conséquences sociétales des nouvelles technologies de l'information et de la communication (TIC), du fait des changements radicaux qu'elles peuvent engendrer dans l'ordre des rapports sociaux. On peut ainsi rappeler le rapport éponyme, remis à la fin des années 1970 au président de la République par Simon Nora et Alain Minc $^{3}$, qui donna lieu aussitôt à un grand nombre de commentaires dont on trouve trace à l'époque dans un article de Jacques Attali ${ }^{4}$.

Aujourd'hui, les craintes associées à la dématérialisation des relations administratives sont certainement amplifiées par l'importance prise par la thématique du non-recours aux droits et aux services au cours de la dernière période. La question de la dématérialisation surgit régulièrement dans les travaux d'étude et de recherche sur le non-recours. C'est

\footnotetext{
1 Plate-forme de l'observation sanitaire et sociale Auvergne-Rhône-Alpes, « Les usages du numérique par les publics fragiles : levier ou frein pour l'accès aux droits ?», Focus de la PFOSS, 29, 2018 ; Amandine Robin, Bérangère Guillet, Marie-Christine Marchand, L'inclusion numérique: entre accès aux droits et risque de fracture, Centre ressources documentaires de l'INSET d'Angers/CNFPT, 2018.

2 Dominique Auverlot, Joël Hamelin et coll., Le fossé numérique en France, Paris, Centre d'analyse stratégique/La Documentation française, 2011 ; Défenseur des droits, Dématérialisation et inégalités d'accès aux services publics, rapport, 2019.

${ }^{3}$ Simon Nora, Alain Minc, L'informatisation de la société, Paris, La Documentation française, 1978.

4 Jacques Attali, «L'informatique et notre avenir », Communication \& Langage, 39, 1978, p. 63-77.
} 
notamment le caractère ambivalent de la dématérialisation qui ressort ${ }^{5}$. Si certains la perçoivent comme un "facilitateur » de l'accès aux droits et aux services, pour d'autres, au contraire, elle est " génératrice » ou « activatrice» de difficultés. C'est en particulier le cas pour les personnes dont l'équilibre de vie passe par la bonne réception de leurs droits ${ }^{6}$. Il apparaît ainsi que les relations administratives dématérialisées nécessaires pour obtenir ou maintenir des droits ouverts ne sont pas porteuses du même enjeu selon les trajectoires de vie. Elles peuvent situer les personnes d'un côté ou de l'autre des frontières de l'intégration et de l'exclusion'.

Ces constats renvoient immédiatement au débat principal sur la «fracture numérique ». Pour autant, ce n'est pas dans cette perspective que nous nous situerons dans cet article pour aborder la relation de la dématérialisation avec le phénomène du non-recours que nous étudions par ailleurs. Si effectivement la dématérialisation des relations administratives peut générer du non-recours, il nous paraît encore plus important de souligner qu'elle ne permet pas d'entendre les personnes qui ne recourent pas ou tout du moins qui ont des difficultés ou bien expriment des résistances - même indépendamment du numérique - pour faire valoir leurs droits. La raison pour laquelle nous proposons cette discussion renvoie à la question suivante: comment prévenir le non-recours, comme le veut l'agenda gouvernemental depuis le Plan de lutte contre la pauvreté et pour l'inclusion sociale de 2013, si les relations en face à face avec les publics disparaissent? La réduction du non-recours est aussi une condition de l'inclusion sociale. C'est donc au travers de la possibilité d'un évitement $\mathrm{du}$ phénomène $\mathrm{du}$ non-recours que nous tenterons de questionner la dématérialisation des relations administratives. Notre propos s'inscrit dans l'une des trois familles de travaux sociologiques sur la fracture numérique, qui porte sur la reproduction des rapports sociaux, à côté des deux autres développées autour des questions des infrastructures et de la socialisation de l'Internet ${ }^{8}$. Nous avons néanmoins besoin de savoir à qui la dématérialisation des relations administratives pose problème avant de nous interroger sur les espaces de relations avec les publics à préserver pour prévenir le non-recours.

\section{À qui la dématérialisation des relations administratives pose-t-elle un problème ?}

La dématérialisation pose un problème à certaines populations à cause du niveau d'autonomie que requiert l'usage du numérique. La difficulté est double. D'une part, il faut avoir accès à un équipement informatique; or c'est loin d'être le cas pour tous. Le Centre d'observation de la société indique dans une note d'étude présentée en décembre 2018 que pas moins de 12,5 millions de personnes, en France, n'ont pas d'ordinateur chez elles et 8 millions pas d'accès fixe par ordinateur à l'Internet ${ }^{9}$. D'autre part, il est indispensable de savoir utiliser un équipement informatique à toutes fins utiles. C'est la question centrale du " pouvoir d'agir par l'informatique », qui avait été formulée dans des termes différents par Nora et Minc. On en comprend aujourd'hui la portée au travers de l'analyse des inégalités liées à la pratique du numérique. De ce point de vue, il est essentiel de considérer deux

\footnotetext{
${ }^{5}$ Brigitte Bouquet, Marcel Jaeger, «L'e-inclusion, un levier? », Vie sociale, 11, 2015, p. 185-192.

6 Pierre Mazet, Héléna Revil, «Vivre en situation de non-recours frictionnel. Enquête dans trois caisses d'allocations familiales », Revue des politiques sociales et familiales, 128, 2018, p. 51-58.

7 Luc Vodoz, "Fracture numérique, fracture sociale : aux frontières de l'intégration et de l'exclusion », SociologieS, 2010, en ligne (https://journals.openedition.org/sociologies/3333).

${ }^{8}$ Fabien Granjon, «Les sociologies de la fracture numérique. Jalons critiques pour une revue de la littérature », Questions de communication, $\quad 6, \quad 2004, \quad$ en ligne (https://journals.openedition.org/questionsdecommunication/4390).

${ }^{9}$ Cette note accessible en ligne, reprend les résultats d'une enquête du CRÉDOC, Baromètre du numérique 2018, $18^{\mathrm{e}}$ édition.
} 
grandes formes de vulnérabilité, comme nous y invite dans un ouvrage récent Marie Garrau ${ }^{10}$. En reprenant la distinction proposée par cette spécialiste de l'étude de la vulnérabilité et de l'autonomie, on peut dire que le manque d'autonomie numérique crée une «vulnérabilité problématique » (car inégalitaire et injuste), appelée « l'illectronisme », au moment même où les personnes concernées cherchent à accéder à des aides pour traiter leurs "vulnérabilités fondamentales » qui méritent réparation ou compensation. Dit autrement, le numérique impose une condition d'autonomie (la « connectivité », ou la capacité à avoir des usagers connectés ${ }^{11}$ ) qui peut constituer une barrière plus ou moins infranchissable dans l'accès aux droits socio-économiques. Alors que les dispositifs de protection sociale et les politiques sociales sont ordonnés de plus en plus autour d'un principe d'individualisation des droits, la dématérialisation des relations administratives, qui s'impose partout, est loin de garantir l'individualisation de l'accès aux droits et de servir la personnalisation des prestations sociales à l'ordre du jour ${ }^{12}$.

L'autonomie numérique est donc un véritable enjeu d'action publique. Aujourd'hui les réponses dépendent pour beaucoup de l'intervention de tiers qui peut être instituée ou pas (par exemple, le recours nécessaire à Emmaüs Connect d'acteurs de la protection sociale, des CAF, CCAS...). En particulier, le bénévolat est mis à contribution pour (r)établir du lien social par la recherche de l'autonomie numérique ${ }^{13}$. Un débat important s'ouvre ici autour du constat, sinon de l'hypothèse, que l'autonomie numérique est un mythe pour les personnes dépendantes de tiers pour réaliser les démarches nécessaires, à partir de l'argument que c'est cette dépendance qui rend vulnérable. Sans entrer dans ce débat qui nous renverrait de nouveau à celui de l'informatisation de la société et, en particulier, au mythe de l'autonomie engendré par les prétentions techno-scientistes ${ }^{14}$, on peut introduire deux remarques. D'une part, il est possible de se demander si la question pourrait être réglée de façon radicale dès lors que l'ouverture et le renouvellement des droits seraient rendus automatiques; ce que le numérique peut faciliter par l'échange de données dématérialisées. Toutefois, l'automaticité qui pourrait réduire mécaniquement la fracture numérique est loin d'être atteinte malgré les projets de simplification des régimes de prestations sociales, souvent justifiés par l'argument d'une réduction (supposée) du non-recours ${ }^{15}$. D'autre part, il faut souligner que le manque d'autonomie numérique ne crée pas ou ne renforce pas systématiquement ou de facto une «vulnérabilité problématique ${ }^{16}$ ». En revanche, c'est le cas lorsque les populations en manque d'autonomie numérique sont aussi celles qui ont le plus besoin de recourir à des aides sociales pour s'en sortir au quotidien. Il convient de s'arrêter un peu sur ce constat parce qu'il permet de caractériser les personnes auxquelles la dématérialisation des relations administratives pose problème.

\footnotetext{
${ }^{10}$ Marie Garrau, Politiques de la vulnérabilité, Paris, CNRS éditions, 2018.

${ }^{11}$ Pierre Mazet, «Vers l'État plateforme. La dématérialisation de la relation administrative », La Vie des idées, mis en ligne le 2 avril 2019.

${ }^{12}$ Christine Cloarec-Le Nabour, Julien Damon, La juste prestation. Pour des prestations et un accompagnement $\begin{array}{lllll}\text { ajustés, } & \text { rapport } & \text { au } & \text { Premier } & 2018\end{array}$ (https://www.gouvernement.fr/sites/default/files/document/document/2018/09/rapport_de_christine_cloarecle nabour_et julien_damon_sur_la juste_prestation.pdf).

${ }^{13}$ Philippe Viallon, « Bénévolat, lien social et numérique », Les Cahiers du numérique, 13, 2017, p. 9-23.

${ }^{14}$ Philippe Bénéton, « Le mythe de l'autonomie », Revue des Deux Mondes, janvier 1997, p. 101-108.

15 Philippe Warin, «Introduction. Scènes d'action contre le non-recours », dans Philippe Warin (sous la direction de), Agir contre le non-recours aux droits sociaux. Scènes et enjeux politiques, Grenoble, Presses universitaires de Grenoble, 2019.

${ }^{16}$ Fabrice Le Guel, « Comment pourrait-on mesurer la double "fracture numérique" ? ", Réseaux, 127-128, 2004, p. 55-82 ; Périne Brotcorne, Gérard Valenduc, «Les compétences numériques et les inégalités dans l'usage d'Internet. Comment réduire ces inégalités ? », Les Cahiers du numérique, 5, 2009, p. 45-68.
} 
L'enquête " Accès aux droits » conduite par le Défenseur des droits en 2016 inclut, dans son module relatif à la relation aux services publics, trois questions sur le numérique à partir desquelles nous pouvons formuler plusieurs constats.

En premier lieu, elle donne quelques indications sur les profils des personnes selon la nature des difficultés qu'elles rencontrent par rapport au numérique. Il ressort notamment que les personnes sans accès à l'Internet sont particulièrement nombreuses parmi les personnes âgées de 60 ans ou plus (22\%) ainsi que parmi celles qui sont les moins diplômées (24\% de celles ayant au plus le BEPC n'ont pas accès à l'Internet) ou encore parmi les personnes handicapées, que le handicap ait ou non fait l'objet d'une reconnaissance administrative. Le profil des personnes trouvant difficilement des informations sur l'Internet dans le cadre de leurs démarches administratives diffère quelque peu. Le niveau de diplôme n'a pas d'impact significatif sur ces difficultés « d'usage »; elles sont en revanche particulièrement fréquentes parmi les plus jeunes ( $21 \%$ des moins de 30 ans sont concernés), parmi les personnes qui s'estiment en situation de handicap, mais qui se sont vu refuser la reconnaissance de ce dernier, ainsi que parmi celles qui sont les plus en difficulté financièrement, qu'elles soient ou non isolées. Ces éléments, et en particulier le dernier constat, signalent que les difficultés d'usage du numérique concernent notamment des personnes dont la situation financière est compliquée.

On peut ajouter que les personnes qui rencontrent des difficultés d'usage du numérique se caractérisent également par la fréquence des situations de renoncement aux soins, de méconnaissance des possibilités de contester les décisions administratives et de discrimination par les administrations. Ainsi $28 \%$ des personnes trouvant difficilement les informations cherchées sur l'Internet ne savent pas qu'elles ont la possibilité de contester les décisions des administrations, $12 \%$ ont déjà été discriminées par une administration et $38 \%$ renoncent, de rarement à très souvent, à des soins de santé. De plus, dans l'enquête du Défenseur des droits, les personnes en difficulté d'usage par rapport à Internet ont un profil comparable à celles dont la relation avec les administrations est déjà compliquée en dehors de toute mention faite du numérique : le «cumul » concerne en particulier les plus précaires et les moins diplômés ${ }^{17}$.

Ces personnes dont les usages du numérique sont fragiles sinon très compliqués déclarent des difficultés financières, se trouvent plus globalement dans des situations de vie précaires et sont plus touchées par le renoncement aux soins. On peut aisément imaginer l'enjeu de pouvoir accéder, autrement que virtuellement, aux services publics pour bénéficier effectivement de leurs droits et l'importance de disposer de possibilités d'échanges avec des agents pour éviter qu'elles n'y recourent pas.

\section{Quelle est la conséquence principale de la dématérialisation ?}

$\mathrm{Au}$ vu de ces résultats, les relations administratives dématérialisées posent problème quand elles privent des personnes qui sont en difficulté pour accéder à leurs droits de la possibilité de pouvoir se tourner vers des agents. En se substituant aux relations directes - en face à face, traditionnelles au guichet, ou par téléphone -, le numérique provoque une " vulnérabilité problématique » car il expose certaines personnes à de nouvelles barrières dans l'accès aux droits. Ce problème est celui qui préoccupe le plus. Cependant, il ne concerne pas l'ensemble de la population car, étonnamment, il passe beaucoup plus inaperçu. En effet, on n'a peut-être pas suffisamment perçu que la conséquence principale de la dématérialisation des relations administratives n'est pas sociale mais sociétale. Le constat, pourtant, est sans

\footnotetext{
${ }^{17}$ Héléna Revil, Christine Olm, Différences d'accès et d'usage du numérique, inégalités et conditions de mise en œuvre de la relation de service aux publics, Paris, La Documentation française, à paraître.
} 
appel puisque nous le vivons tous: le déploiement en force du numérique met fin massivement et brusquement aux relations directes avec les agents prestataires auxquelles nous avons tous été habitués jusque-là. Nous assistons de cette façon, plutôt passivement, à une rupture épistémique au sein de la société. Ces relations directes, celles au guichet la plupart du temps, ont contribué, à côté des relations professionnelles, syndicales et associatives, à structurer depuis longtemps l'organisation des rapports sociaux. Or ces relations disparaissent, en même temps que les relations professionnelles se transforment et que la désyndicalisation s'accroît, à l'exception de la vie associative qui reste vivace et se renouvelle. Si l'on accepte la notion d'«homéostasie sociale» proposée par Norbert Wiener $^{18}$, on pourrait même se demander, plus loin encore, si la dématérialisation des relations administratives ne constitue pas une transformation en profondeur des modalités qui ont permis de stabiliser l'ordre social en plaçant de façon directe les citoyens dans « la main gauche de l'État ${ }^{19}$ ».

En tout cas, les signaux ne manquent pas. Le numérique rendrait illisible, dans certains cas, la relation aux administrations, organismes sociaux et services publics, dans la mesure où l'on demande à leurs agents de jouer sur deux plans, par la relation humaine et par la relation numérique, et de renvoyer vers la seconde les usagers à qui on ne sait pas/on ne veut pas répondre. Dans d'autres cas, il irait même jusqu'à vider les relations administratives de leur « efficacité identitaire» dès lors qu'il éradique (à cause de son objectif d'économie de moyens) le va-et-vient interprétatif que seuls les échanges directs avec des agents humains permettent. Ces échanges entre personnes, agents et usagers, à des places et dans des rôles différents, nourrissent en effet des rapports de citoyenneté au sens où les interactions engagent des représentations et des attentes sur les normes sociales qui sont en jeu. C'est ce que nous a appris l'étude des relations de service ${ }^{20}$, que l'approche de la réception des politiques par les publics vient élargir aujourd'hui avec beaucoup d'intérêt ${ }^{21}$. C'est aussi le cas de celle dite des «policy feedbacks » qui mesure (par les grandes enquêtes sur les valeurs) la confiance dans les institutions par des questions sur les conséquences des expériences individuelles des politiques publiques et des rapports avec les organisations prestataires ${ }^{22}$. En piochant dans tous ces travaux, on en ressortirait de multiples constats sur le fait que la dématérialisation supprime une myriade de relations administratives à partir desquelles chacun nourrit satisfait ou excédé - le sentiment de faire société.

Il faut toutefois se garder de faire du passage au numérique la seule, voire la principale, explication du déclin des relations directes avec les usagers comme modèle d'administration, dont le guichet serait l'emblème. La relation au guichet, qui a gagné en importance pendant toute une période (années 1980-1990) dans les politiques de modernisation de l'administration et des services publics, a perdu progressivement ses moyens du fait de la marchandisation croissante du social et des restrictions budgétaires successives qui dégradent les conditions de travail des agents prestataires ${ }^{23}$. Aussi, la

\footnotetext{
18 Ronan Le Roux, «L'homéostasie sociale selon Norbert Wiener », Revue d'histoire des sciences humaines, 16, 2007, p. 113-145.

19 Jean-Claude Monod, «Les deux mains de l'État. Remarques sur la sociologie de la misère de Pierre Bourdieu », Esprit, 214, 1995, p. 156-171.

20 Philippe Warin, «Les "ressortissants" dans les analyses des politiques publiques », Revue française de science politique, 49 (1), 1999, p. 103-121.

21 Anne Revillard, "Saisir les conséquences d'une politique à partir de ses ressortissants : la réception de l'action publique », Revue française de science politique, 68 (3), 2018, p. 469-491.

22 Alexis Spire, «État des lieux. Les policy feedbacks et le rapport ordinaire à l'État», Gouvernement et action publique, 4 (4), 2016, p. 141-156.

${ }^{23}$ Michel Chauvière, Trop de gestion tue le social. Essai sur une discrète chalandisation, Paris, La Découverte, 2007 ; Françoise Dreyfus, « Personnels et usagers des services publics : des relations transformées ? », Regards
} 
dématérialisation des relations administratives se substitue-t-elle de plus en plus au modèle du guichet dans la mesure où elle répond à un objectif général d'efficience accrue des dépenses publiques. Elle a sous cet angle l'avantage d'être une solution particulièrement efficace - en tout cas à court terme - pour éviter les coûts de production induits par des échanges imprévisibles, pour partie liés aux demandes complexes, qui ont conduit à parler d'un « débordement du social» au guichet ${ }^{24}$.

En attendant que la révolution digitale donne un jour aux ordinateurs des administrations, des organismes sociaux et des services publics une intelligence comparable à la réflexivité humaine, la dématérialisation crée donc, par un vide de relations personnifiées, un changement radical dans les processus courants de démarches administratives. Une transformation s'impose donc dans les rapports concrets des citoyens à l'action publique. Pourtant, des travaux ont montré que face aux inégalités sociales, tout en adhérant massivement au principe de neutralité du service public, la plupart des agents prestataires ont intériorisé que les « ressortissants » - les individus ou les groupes à qui les politiques sont destinées ${ }^{25}$ - ne sont pas à égalité de chances et que la réussite des relations avec eux réside d'abord dans la possibilité d'un examen approfondi des demandes, même au prix d'une pression accrue dans le travail ${ }^{26}$. Aussi, en transformant les relations aux ressortissants, le numérique pose-t-il très fortement aujourd'hui la question des espaces à préserver pour que les professionnels des différents métiers de la santé et du social aient encore la possibilité d'intervenir directement auprès des personnes selon les besoins.

\section{Quels espaces de relation avec les publics faut-il préserver pour prévenir le non- recours?}

La Stratégie de prévention et de lutte contre la pauvreté présentée par le président de la République le 13 septembre 2018 apporte probablement des premiers éléments de réponse, en particulier dans son objectif central de renforcement et de valorisation du travail social. En l'espèce, les orientations qui ont donné lieu à la mise en place de groupes de travail en vue d'élaborer des propositions portent sur les usages du numérique par les travailleurs sociaux, mais aussi sur les pratiques d'intervention collective, la participation des personnes accompagnées, le premier accueil social inconditionnel et «l'aller-vers ». En attendant de savoir ce que seront ces propositions, et surtout celles qui seront retenues, les premières orientations conduisent plutôt vers l'hypothèse d'un modèle général d'administration du social dans lequel le travail social aurait pour mission principale d'accompagner celles et ceux qui n'ont pas l'autonomie nécessaire pour accéder à leurs droits.

La demande de propositions pour faciliter les usages du numérique par les travailleurs sociaux vise en particulier à garantir une maîtrise suffisante du numérique chez les professionnels pour qu'ils puissent aider au mieux celles et ceux qui rencontrent des difficultés dans l'accès de plus en plus dématérialisé aux droits. Les autres groupes travaillent sur des thématiques qui consistent à préparer autrement l'intervention sociale destinée à accompagner des populations en situation de précarité, ou à les sortir des zones de non-

croisés sur l'économie, 2, 2007, p. 246-254; Annie Dussuet, Laura Nirello, Emmanuelle Puissant, « De la restriction des budgets des politiques sociales à la dégradation des conditions de travail dans le secteur médicosocial », Revue de l'IRES, 91-92, 2017, p. 185-211.

${ }^{24}$ Vincent Dubois, La vie au guichet. Relation administrative et traitement de la misère, Paris, Economica, 2010 .

25 Julien Lévy, Philippe Warin, « Ressortissants », dans Laurie Boussaguet, Sophie Jacquot, Pauline Ravinet (sous la direction de), Dictionnaire des politiques publiques, Paris, Presses de Science Po, 2019.

${ }^{26}$ Philippe Warin, Les dépanneurs de justice. Les "petits fonctionnaires 》 entre qualité et équité, Paris, LGDJ, 2002. 
demande en ce qui concerne «l'aller-vers» des populations qui échappent aux radars institutionnels. Les orientations fixées prédéfinissent en quelque sorte des espaces de relations directes à préserver, alors que la dématérialisation des relations administratives conduit peu à peu à la suppression des guichets. On passe à des techniques d'intervention sociale ciblées sur des populations qui dépendent de la bonne réception de leurs droits et qui peuvent être désarçonnées par la dématérialisation des relations administratives mais aussi du travail social $^{27}$. Des espaces, comme ceux du premier accueil inconditionnel, de «l'aller-vers », de la participation et des interventions collectives, préserveraient donc la possibilité d'intervenir en direct auprès de populations en particulier selon les besoins.

Il est possible que ces «nouveaux espaces » du travail social aident à traiter la question de la fracture numérique telle qu'on l'entend habituellement. En effet, ils peuvent contribuer à activer l'inclusion sociale de deux façons. De façon pratique, par les relations directes qu'ils créent nécessairement entre agents et usagers pour apporter des réponses aux «vulnérabilités fondamentales" des personnes, mais également pour lever les barrières créées par les relations dématérialisées. De façon symbolique, parce que ce sont des espaces discursifs qui compensent le caractère disruptif du numérique (qui met fin au va-et-vient interprétatif que seuls des échanges directs, notamment en face à face, permettent) et qui peuvent aussi aider à trouver l'assentiment des ressortissants.

Toutefois, la question de la fracture numérique n'est pas seulement celle de l'autonomie numérique et la réponse n'est pas simplement celle de la littératie numérique. Le problème n'est en effet pas uniquement celui des « individus défaillants » ou plus exactement celui de l'inclusion sociale d'une population moins autonome. Le problème principal que pose la dématérialisation des relations administratives et du travail social, outre de réduire les espaces et les temps pour des relations individuelles en face à face ou par téléphone, est aussi collectif et fondamentalement politique. La raison en est simple : si la dématérialisation n'empêche pas de refuser ce qui est proposé (en particulier, les populations «autonomes » peuvent toujours abandonner une session de demande d'ouverture de droits sur l'Internet), ce qui donne au numérique une vertu démocratique, en revanche elle ne permet plus au ressortissant - quel qu'il soit - d'expliquer son refus et surtout de demander une justification au prestataire. Puisque la conséquence principale de la dématérialisation des relations administratives est de réduire sinon de supprimer un vaste espace de relations sociales directes, les ressortissants ne sont plus en mesure de poser directement des questions à des agents pour bien comprendre ce qui leur est proposé, les conditions pour en bénéficier, et donc in fine cela ne leur permet pas/plus d'avoir toutes les cartes en main pour décider s'ils acceptent ou non de " prendre » ce qui leur est proposé. Dans ce cas, le numérique peut aussi être une manière de " botter en touche», de se débarrasser de la complexité : on renvoie l'allocataire, l'assuré, l'usager vers des espaces numériques (Caf.fr, compte Améli) et on lui « enjoint » de comprendre tout seul, de chercher les infos par lui-même ${ }^{28}$. Ainsi, le numérique amorce-t-il un retrait d'ampleur par des administrations, des organismes sociaux et des services publics de la relation personnifiée, d'autant que les échanges de données entre organisations préparent la disparition du système déclaratif qui constituait la pierre angulaire de la plupart des démarches administratives.

Pour bien comprendre le jeu entre dématérialisation et non-recours, il est alors indispensable de dire que le problème n'est pas seulement - ni même principalement si l'on se situe sur le plan de la démocratie - celui de barrières supplémentaires dans l'accès aux droits, mais également - sinon surtout - celui de la suppression d'un vaste espace (celui des

\footnotetext{
27 Joran Le Gall, Cristina De Robertis (sous la direction de), «Le travail social à l'épreuve du numérique », Revue française de service social, 264, 2017.

${ }^{28}$ Pierre Mazet, Héléna Revil, « Vivre en situation de non-recours frictionnel... », op. cit.
} 
relations en face à face) où il est possible d'exprimer et de comprendre les raisons d'un nonrecours dans un grand nombre de situations. En effet, les plates-formes numériques par lesquelles il faut passer pour déposer et traiter une demande traitent tout visiteur comme un consommateur à qui il resterait un choix unique, celui de prendre ou de laisser, de continuer ou d'abandonner... puisqu'il n'a plus d'interlocuteur pour l'informer et l'aider dans sa démarche.

Le jeu, actuellement, pour beaucoup d'administrations, d'organismes sociaux et de services publics consiste donc à « maintenir » pour une gestion attentionnée des situations, qui peuvent être très nombreuses, la possibilité d'une relation directe (en organisant des rendez-vous des droits pour certains usagers, en mettant en place des dispositifs comme les plates-formes d'intervention départementales pour l'accès aux soins et à la santé - PFIDASS etc.), et à l'évacuer dans le traitement en masse des demandes courantes. Pour l'instant, le numérique est une manière de limiter le plus possible les relations directes à certains types de situations.

Mais ensuite, qu'en sera-t-il lorsque $100 \%$ des services publics seront dématérialisés ? En allant jusqu'à cette extrémité, la dématérialisation pourrait introduire un bouleversement dans les rapports sociaux. Si les ressortissants n'ont plus d'interlocuteurs humains, sont-ils encore des citoyens envers qui les administrations, organismes sociaux et services publics sont redevables et doivent se justifier ? Et que reste-t-il alors du principe du droit de créance qui a fondé notre modèle d'administration et de service public ? Parler de mutation des rapports sociaux ne nous paraît pas exagéré. Cette hypothèse est d'autant plus plausible que le numérique n'en serait que l'une des modalités. D'autres évolutions peuvent également être prises en compte. Par exemple, si l'on ose un parallèle avec le prélèvement de l'impôt à la source (possible grâce à la dématérialisation des données et à leur circulation entre centres de traitement), on peut alors introduire un autre changement administratif récent et développer l'idée selon laquelle on assisterait sur différents plans à une transformation en profondeur de la citoyenneté. Le citoyen, dans le cas de la e-administration, n'est plus le « référent ultime du service public » auquel il faut répondre, mais un producteur du service à rendre, dans le cas du prélèvement de l'impôt à la source. Il n'est plus actionnaire d'un État producteur mais débiteur d'un État qui exige le paiement d'une créance nécessaire à son action ${ }^{29}$.

\section{Conclusion}

En s'interrogeant sur la nécessité de préserver des espaces de relations directes et personnalisées pour prévenir le non-recours, on pose la question de l'inclusion sociale sous l'angle des rapports sociaux qui lui sont nécessaires, que la dématérialisation des relations administratives contribue à faire disparaître. Ces espaces de relations directes sont indispensables pour accompagner vers leurs droits les personnes en difficulté pour y accéder ; ils permettent notamment d'éviter tant que possible l'exclusion numérique. Pour autant, les restreindre au seul besoin d'une intervention sociale ciblée conduit à n'écouter que ceux qui ne peuvent pas accéder à leurs droits et à oublier largement tous ceux qui ne veulent plus (pour une raison ou pour une autre) les faire valoir. Aussi, les espaces de relations directes, qui se dessinent en même temps que se redéfinissent les fonctions du travail social, pourraient-ils laisser de côté tout un pan du phénomène du non-recours, autour de la nondemande raisonnée ou volontaire ${ }^{30}$. En même temps, les espaces qui seraient ainsi préservés

\footnotetext{
${ }^{29}$ Hubert Étienne, « Comment la fiscalité est devenue illégitime », Le Monde, 13-14 janvier 2019, p. 6.

${ }^{30}$ Philippe Warin, Le non-recours aux politiques sociales, Grenoble, Presses universitaires de Grenoble, 2016 ; Héléna Revil, Philippe Warin, «Non-recours », dans Laurie Boussaguet et coll. (sous la direction de), Dictionnaire des politiques publiques, op. cit.
} 
ont leur importance, du fait de l'épuisement généralisé des missions exercées au guichet sous les effets de la suppression de bon nombre de services en proximité des populations.

Retour à l'histoire donc, puisque l'on retrouve la question centrale qui avait prévalu dans la recherche sur les relations de service développée dans les années 1980-1990 pour accompagner les politiques de modernisation de l'administration et des services publics : celle de « l'autolimitation de la puissance publique », selon la formule du sociologue Isaac Joseph qui fut le coordinateur scientifique d'un séminaire de recherche et d'un colloque qui firent date sur "La relation de service dans le secteur public». Cette autolimitation n'est pas seulement un devoir ou une option de la prudence démocratique, mais une condition pragmatique de son exercice ${ }^{31}$. On pensait l'obtenir en favorisant la participation des usagers, mais les choses ont peu bougé sur ce plan malgré les discours. On retrouve cette exigence, trente ans plus tard, avec la thématique du non-recours qui, bien que posée sur l'agenda gouvernemental, pourrait être singulièrement édulcorée si le modèle en expansion de l'eadministration ne laissait plus d'espaces à l'expression directe des raisons qui amènent bon nombre de personnes à ne pas ou plus recourir à leurs droits, ou à désespérer de ne pas pouvoir y accéder.

\footnotetext{
${ }^{31}$ Isaac Joseph, « Le service public et ses usagers », dans DRAST/CPVST, La production de l'assentiment dans les politiques publiques. Actes des ateliers-conférences du GRASS organisés à l'IRESCO en 1991-1992, 1993, p. 8592.
} 